Journal of Innovative Optical Health Sciences

Vol. 12, No. 2 (2019) 1950006 (10 pages)

(C) The Author(s)

DOI: $10.1142 / \mathrm{S} 1793545819500068$

\title{
Enhancement of microvessel in laser speckle image using gaussian kernel template
}

\author{
Yameng Zhang, Yuemei Zhao, Weitao Li*, Zhiyu Qian ${ }^{\dagger}$ and Lidong Xing \\ Department of Biomedical Engineering \\ Nanjing University of Aeronautics and Astronautics \\ 211106, Nanjing, P. R. China \\ *liweitao@nuaa.edu.cn \\ †zhiyu@nuaa.edu.cn
}

Received 23 October 2018

Accepted 9 January 2019

Published 27 February 2019

\begin{abstract}
Laser speckle contrast imaging (LSCI) is an optical imaging method, which can monitor microvascular flow variation directly without addition of any ectogenous dye. All the existing laser speckle contrast analysis (LASCA) methods are a combination of spatial and temporal statistics. In this study, we have proposed a new method, Gaussian kernel laser speckle contrast analysis (gLASCA), which processes the raw images primarily with the Gaussian kernel operator along the spatial direction of blood flow. We explored the properties of gLASCA in the simulation and animal cerebral ischemia perfusion model. Compared with the other existing speckle processing methods based on spatial, temporal, spatial-temporal or anisotropic linear structure; the present gLASCA method has a high spatial-temporal resolution to respond the change of velocity especially in microvasculature. Besides, the gLASCA method obtains approximately $10.2 \%$ and 7.1\% higher contrast-to-noise ratio (CNR) over the anisotropic linear method (aLASCA) in the simulation and experiment models. For these advantages, gLASCA could be a better method for local microvascular laser speckle imaging in terms of cerebral ischemia reperfusion, spreading depression and brain injury diseases.
\end{abstract}

Keywords: Brain vasculature; blood flow; contrast imaging; linear operator; contrast-to-noise ratio.

\section{Introduction}

In recent years, laser speckle contrast imaging (LSCI) has become an emerging method of optical vascular flow imaging. ${ }^{1}$ Compared with brain functional imaging methods, such as near infrared spectroscopy imaging and laser Doppler, LSCI has a higher temporal resolution. Moreover, LSCI is a non-invasive method without the introduction of any exogenous dyes in consideration of angiography and fluorescence imaging. ${ }^{2}$ LSCI can simultaneously monitor the cerebral cortex of vascular morphology and blood flow velocity. ${ }^{3}$ At present, the contrast value $(K)$ in laser speckle contrast analysis (LASCA) is used to study capillary blood flow, ${ }^{4}$

${ }^{*}, \dagger$ Corresponding authors.

This is an Open Access article published by World Scientific Publishing Company. It is distributed under the terms of the Creative Commons Attribution 4.0 (CC-BY) License. Further distribution of this work is permitted, provided the original work is properly cited. 
retinal blood flow $^{5}$ and cerebral blood flow (CBF) ${ }^{6,7}$

Scattering laser reflected from a rough surface with random interference leads to the generation of speckle pattern, which is spatially blurred due to the movement of scattering particles. Definition $K$ of speckle contrast in a local region can be expressed as the quotient of the standard deviation value to the mean value of the light intensity, $K=\sigma / \mu$. Traditionally, $K$ is calculated in the spatial domain of laser speckle spatial contrast analysis (LSSCA) ${ }^{8}$ or the temporal domain of laser speckle temporal contrast analysis (LSTCA). ${ }^{9}$ The spatial resolution and temporal resolution are compromising with each other. Many studies have reported that the isotropic approach could address the issue of spatiotemporal resolution.

Spatially derived contrast using LASCA (sLASCA) proposed by Briers and Webster, ${ }^{10}$ is an enhancement of the basic LSSCA technique. In sLASCA scheme, the derived contrast value can be obtained by averaging over temporal frames of raw speckle images, which is different from LSSCA. Temporally derived contrast using LASCA (tLASCA) also focuses on the statistical temporal frames. ${ }^{11}$ For example, in tLASCA method, the parameter $n$ means the number of temporal frames, usually the total convolutional window selected is (3) $(3)(n / 2)$ where $3 \times 3$ pixel is the spatial window. Both methods would smooth LSSCA or LSTCA images in the spatial or temporal domains to obtain the degree of robustness. Furthermore, in spatialtemporal cuboid LASCA (stLASCA) ${ }^{12}$ method, a cuboid of pixels can be used to calculate the local $K$ for achieving the flexible and quantitative scheme in spatiotemporal domain. Since speckle blurring of blood flow can show directional sensitivity, anisotropic LASCA ${ }^{13}$ (aLASCA) proposed by Abhishek focuses on the anisotropic spatial neighborhood and calculates linearly local speckle contrast. aLASCA not only improved microvascular distinguishability, but also strengthened the local noise.

Considering the advantages and flaws in all the methods, we introduced a Gaussian kernel LASCA (gLASCA) method of matched operator to acquire the local speckle contrast along the estimated vascular direction. Then, we performed the simulation and mice experiments to investigate and assess the performance of this algorithm. Comparing with the other existing speckle processing methods based on spatial, temporal, spatial-temporal and anisotropic linear structure, the present method improved the contrast-to-noise ratio and had a high spatialtemporal resolution in microvasculature. Meanwhile, the matched operator in gLASCA method also decreased the local noise and smoothed the main branches of blood flow in processed images.

\section{Methods and Experiment}

\subsection{Simulation of time integrated dynamic speckle image}

The time-integrated dynamic speckle images from "copular" theory ${ }^{14}$ is described as Eq. (1).

$$
\left\{\begin{array}{l}
Z(k)=\sqrt{-2 \ln X_{1}} \cos \left(2 \pi X_{2}+\frac{\pi}{2} \cdot \frac{k-1}{n-1}\right) \\
T(k)=\mathrm{CDF}[Z(k)] \\
\varphi(k)=I \cdot e^{2 \pi m T(k) i}
\end{array}\right.
$$

where $X_{1}$ and $X_{2}$ are two independent random variables in statistics which obey the uniform distribution in the unit interval; $n$ is the length of the sequence, and $k=1,2, \ldots, n$. Therefore, we set $n$ to 50 , and set $X_{1}$ and $X_{2}$ to $256 \times 256$ pixels, respectively. Then, we could obtain a series of $Z(k)$ complying with Gaussian distribution. Through normal cumulative distribution function, we could obtain a correlated sequence of $T(k)$. Moreover, we created 50 frames correlated static laser speckle images by performing Fast Fourier Transformation (FFT). Here, $I$ is intensity image of scatter surface, $2 \pi m T(k)$ is the phase of $\varphi(k)$, and $n$ is a multiplicative factor to simulate varied velocity by controlling the correlation degree. Finally, we could get a frame of dynamic laser speckle image by averaging over the consecutive 20 frames of the static laser speckle images. In the simulation, the minimum speckle size was set as the twice the size of the detector pixel to satisfy Nyquist criterion with box-car convolution filters. The frames of dynamic laser speckle images were $30 .{ }^{15}$ To explore the effectiveness of the multiplicative factor $m$ in Eq. (1), we simulated different flow rate of time-integrated dynamic speckle images by changing the value of $5,10,15,20$ and 25 .

\subsection{Experimental setup}

Figure 1 shows the schematic diagram of the LSCI system. A He-Ne laser $(632.8 \mathrm{~nm}$ and $3 \mathrm{~mW}$, SPLHN250R, Hangzhou SPL Photonics, China) is adjusted by a collimator. Then the laser passes an 


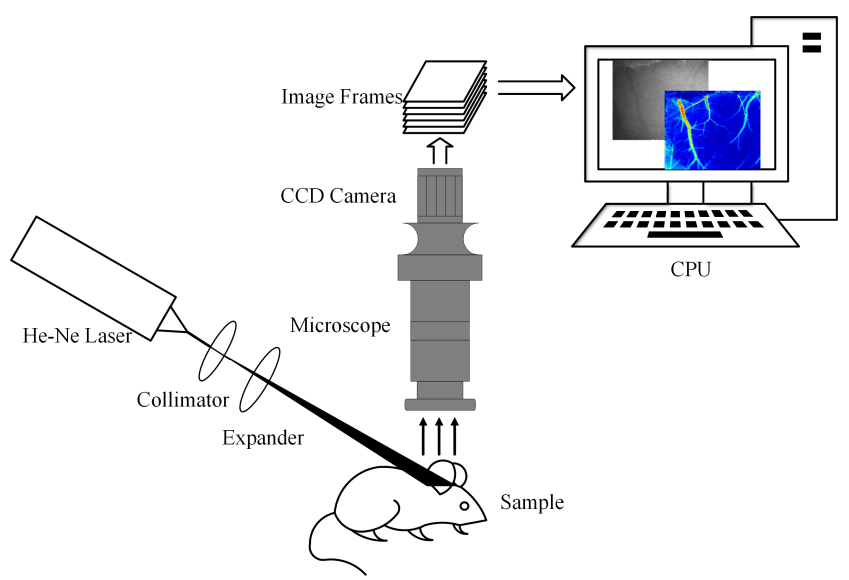

Fig. 1. Schematic of LSCI system.

expander and irradiates the imaging region with a diameter of $\sim 12 \mathrm{~mm}$ and the incident angle of $30-45^{\circ}$. The beam expander works at $450-680 \mathrm{~nm}$. Meanwhile, the image of illuminated region is magnified by a zoom stereo microscope (50486A, Navitar, USA) and captured by a monochrome 12 bit CCD camera (GS3-U3-51S5M-C, Point Grey, Canada, $3.45 \mu \mathrm{m} \times 3.45 \mu \mathrm{m}$ per pixel) with $2448 \times$ 2048 pixels. The exposure time of the CCD was $15 \mathrm{~ms}$. Images are acquired through the programming software at $20 \mathrm{~Hz}$. Then, a stack of 30 raw images is stored and processed using MATLAB routines (Mathworks, MA, US).

\subsection{Animal preparation}

All animal experiments were conducted in accordance with the guidelines of the Institutional Animal Care and Use Committee at Nanjing University of Aeronautics and Astronautics. The Institute of Cancer Research (ICR) mice were purchased from the Qinglongshan Animal Experiment Center (Nanjing, China) and housed in a cage for 7 days after individualization (postnatal day [PD] 36).

Five ICR mice were anesthetized by using $5 \%$ chloral hydrate $(400 \mathrm{mg} / \mathrm{kg}$, i.p.) and placed in a stereotaxic frame with skull-reduced flat orientation. The skin was removed and a cranial window (about $2 \mathrm{~mm} \times 2.5 \mathrm{~mm}$ ) was drilled right side of midline overlaying the parietal cortex. The imaging acquisition was performed on the cranial window after the end of the surgery and temperature was continuously measured.

In this study, a transient middle cerebral artery (MCA) occlusion model was induced as reported protocol. ${ }^{16}$ A small micro-clip was placed tenderly on the most proximal branch of one side of the distal carotid arteries. After $20 \mathrm{~min}$, the micro-clip was removed and both carotid sutures were released to allow for reperfusion. Body temperature was maintained at $37.8^{\circ} \mathrm{C}$ by a heat lamp during surgery and for $2 \mathrm{~h}$ after the start of reperfusion.

\subsection{Design of matched operator}

Microvessels had several small curves. The antiparallel pairs could be approximated by piecewise linear segments. ${ }^{17,18} \mathrm{We}$ utilized a more effective method and selected the suitable line operator. ${ }^{16}$ The minimal value of contrast sum $D(i, j)$ is defined as Eq. (2)

$$
\min (D(i, j))=\sum_{P \in\left\{\left\|(i, j)-\left(i_{0}, j_{0}\right)\right\| \leq \delta\right\}} K_{P(i, j)},
$$

where $K_{P(i, j)}$ is evaluated along lines of fixed length passing through the target pixel $P\left(i_{0,} j_{0}\right)$ at different orientations. The candidate matched model is the constant $K$ image obtained by processing raw speckle frames in LSTCA. Furthermore, the line detector is illustrated in Fig. 2. We considered 12 directions with the interval of $15^{\circ}$. Since the optimal spatial direction is the direction of minimal contrast sum in regard to the central pixel $P\left(i_{0}, j_{0}\right), \delta$ depends on the length of the pixel. Usually the diameters of vessels the are approximately less than or equal to 15 pixels $(\leq 100 \mu \mathrm{m})$. Therefore, 9 pixels line operator could be selected as structuring element to perform morphological operations.

Furthermore, the vascular width decreased while it travelled radially outward from main vein and aorta, such variation of vessel caliber was taper, which might be estimated by a Gaussian curve. ${ }^{19}$ When the definition of matched filter is extended to two-dimensional images, the convolution kernel will fluctuate by subtracting the average of Gaussian curve from the literature. ${ }^{20}$ This kernel could be mathematically expressed as Eq. (3).

$$
W=-e^{\left(-x^{2} / 2 \sigma^{2}\right)} \quad \text { for }|y| \leq L / 2,
$$

where $L$ is the length of the segment direction which is to be aligned with the $y$-axis as an assumption. $\sigma$ defines as the spread of the intensity profile. Considering the Gaussian kernel is an unlimited extension, it needs to discard the points far from the center point. When $L=9$ and $\sigma=1$, the procedure 


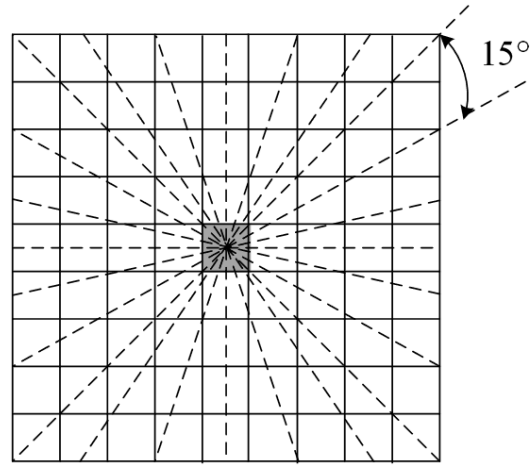

(a)

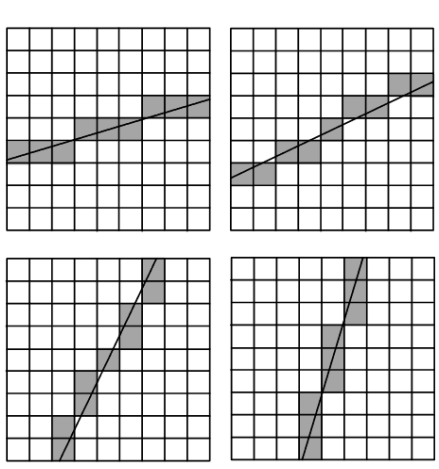

(b)

Fig. 2. (a) 12 directions to assess line intensity of shaded pixel. (b) lines of 9 pixels with direction: $15^{\circ}$ (top left), $30^{\circ}$ (top right), $60^{\circ}$ (bottom left), and $75^{\circ}$ (bottom right).

could suppress significantly the response induced by the noise in the background contrast value in Fig. $3 .^{21}$

For the vessels at different orientations, the kernel should be rotated accordingly. The rotation matrix is given by Eq. (4).

$$
r_{i}=\left[\begin{array}{cc}
\cos \theta_{i} & -\sin \theta_{i} \\
\sin \theta_{i} & \cos \theta_{i}
\end{array}\right]
$$

where $\theta_{i}$ is the direction of the $i$ th Gaussian kernel function and matches the cerebral vessel. It is assumed that the angular resolution is $15^{\circ}$. The optimal direction of mask in Eq. (3) has been defined in Eq. (2).

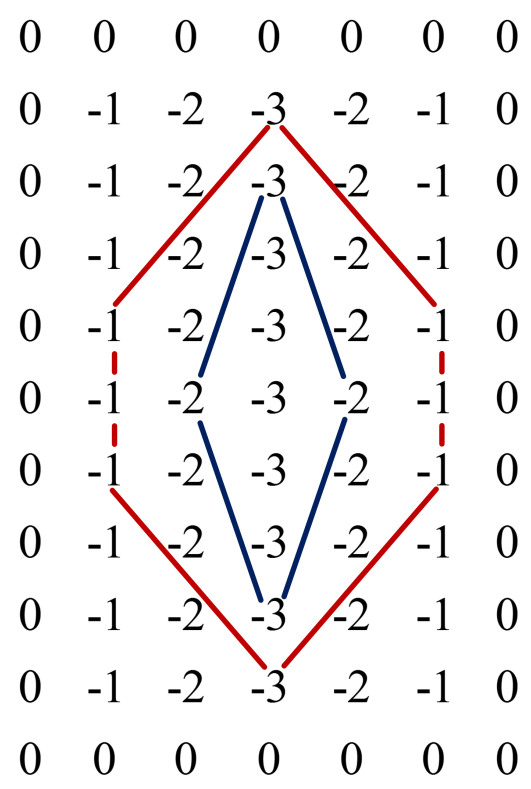

Fig. 3. The Gauss kernel that has been to match the segment along the vessel direction. Here the region blue line circled corresponds to a $3 \times 3$ spatial window and the red line circled corresponds to a $5 \times 5$ spatial window.
If $W_{i}\left(x_{i}, y_{i}\right)$ denotes the value in the window of Gaussian kernel, the weight coefficient $\left(m_{i}\right)$ of Gaussian kernel function is described as Eq. (5).

$$
m_{i}\left(x_{i}, y_{i}\right)=W_{i}\left(x_{i}, y_{i}\right)=/\left(\sum_{\left(x_{i}, y_{i}\right) \in N} W_{i}\left(x_{i}, y_{i}\right)\right) \text {, }
$$

where $N$ is defined as specific region of Gaussian kernel seen in Fig. 3. Thus, the value $W_{i}^{\prime}$ in the convolution mask of this algorithm is given by Eq. (6) because the mean and variance in the speckle images need to be considered in the calculation of the weight coefficient in the kernel.

$$
W_{i}^{\prime}\left(x_{i}, y_{i}\right)=W_{i}\left(x_{i}, y_{i}\right) \cdot m_{i}\left(x_{i}, y_{i}\right)
$$

Contrast-to-noise ratio $(\mathrm{CNR})^{1}$ is calculated for each vascular region to evaluate all algorithms of LSCI as follows.

$$
\mathrm{CNR}=\frac{\mu_{\text {tissue }}-\mu_{\text {background }}}{\sigma_{\text {background }}},
$$

where $\mu_{\text {tissue }}$ and $\mu_{\text {background }}$ are the mean of speckle contrast on vessels and background regions independently; meanwhile, $\sigma_{\text {background }}$ is the variance of speckle contrast values on background cerebral region. Variance of vascular pixel intensities was considered as the flow dependent signal because it contained useful information and excluded from the denominator. ${ }^{22}$

\section{Results}

gLASCA showed a better performance for in vivo imaging of simulated dynamic speckle images 
Table 1. Parameters involved in comparison of various speckle processing methods for uniformity (NA: not applicable).

\begin{tabular}{lccc}
\hline Processing method & $\begin{array}{c}\text { Spatial convolution window } \\
(N s) \text { (simulation/mouse })\end{array}$ & $\begin{array}{c}\text { Temporal frames }(N) \\
(\text { simulation/mouse })\end{array}$ & $\begin{array}{c}\text { Temporal convolution window } \\
(N t)(\text { simulation/mouse })\end{array}$ \\
\hline LSSCA & $3 \times 3 / 5 \times 5$ & $1 / 1$ & NA/NA \\
LSTCA & $1 \times 1 / 1 \times 1$ & $30 / 30$ & NA $/ \mathrm{NA}$ \\
tLASCA & $3 \times 3 / 5 \times 5$ & $30 / 30$ & $(\mathrm{~N} / 2) /(\mathrm{N} / 2)$ \\
sLASCA & $3 \times 3 / 5 \times 5$ & $30 / 30$ & $\mathrm{NA} / \mathrm{NA}$ \\
stLASCA & $3 \times 3 / 5 \times 5$ & $30 / 30$ & $5 / 5$ \\
aLASCA & $9 \times 1 / 25 \times 1$ & $30 / 30$ & $5 / 5$ \\
gLASCA & Linear operator (same counts) & $30 / 30$ & $5 / 5$ \\
\hline
\end{tabular}

comparing with other five representative processing methods including LSSCA, LSTCA, tLASCA, sLASCA, and stLASCA. Table 1 shows the neighborhood parameters in each algorithm. The spatial and temporal resolution were the key factors for choosing appropriate parameters (Table 1). Both the processing frames of simulation and experiments speckle images were 30 frames in different conditions except in LSSCA method. Thus, we selected 5 frames $(N t)$ as temporal convolution window aimed at the spatialtemporal convolution algorithms. Additionally, $\mathrm{Ns}$ shown in Table 1 ensured a fair comparison among these 6 algorithms. Since neither spatial resolution of speckle images in simulation model nor mouse experiment was identical, $N s$ need to be selected on the basis of pixel size, which means that the higher image resolution would correspond to the (a)

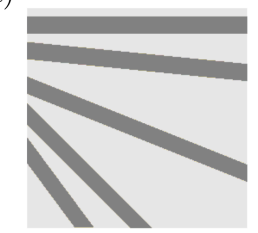

(b)

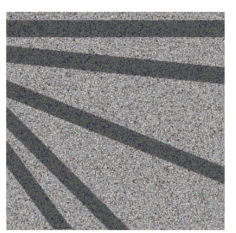

(f)

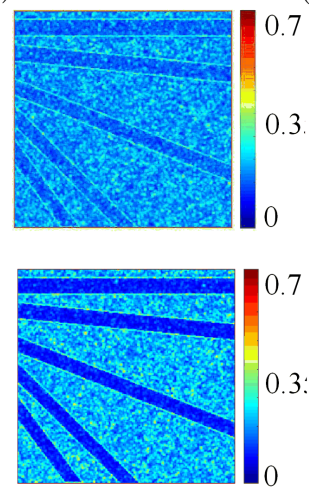

(c)
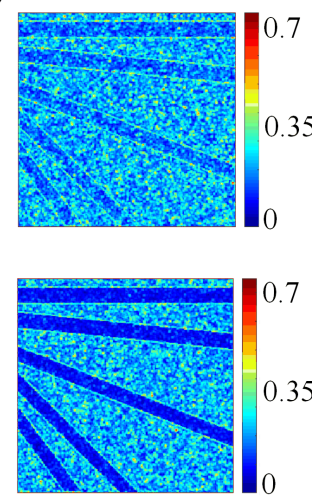

$(\mathrm{g})$

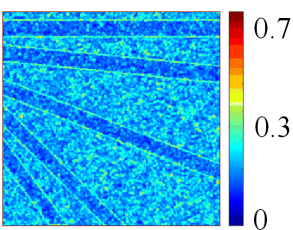

(d)
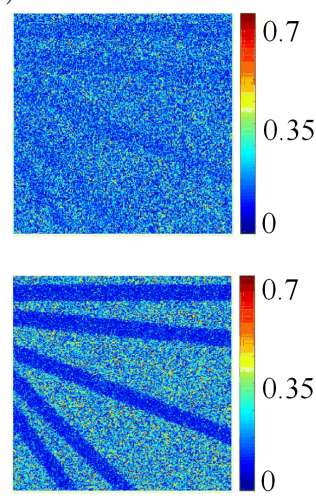

(h)

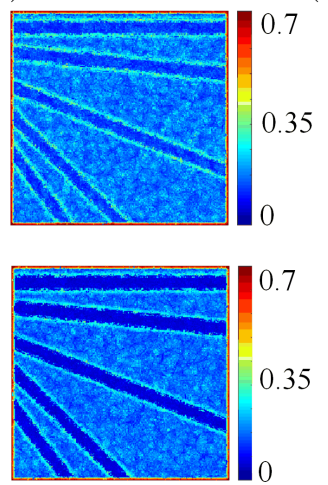

(e)
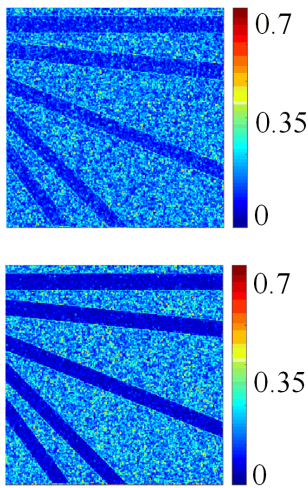

(i)
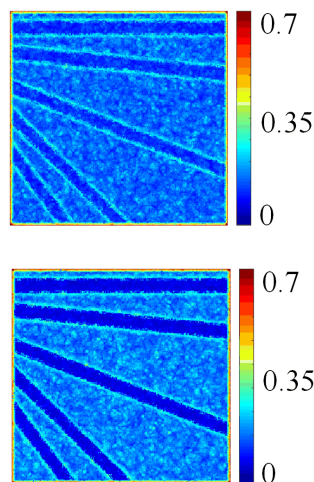

Fig. 4. (a) Intensity image of scattering surface. Dark region represent blood vessels and the gray value is constant. (b) Simulated time-integrated dynamic speckle images with "copular" theory. Comparisons between contrast images computed from the simulated speckle images with parameter $m$ using (c) LSSCA, (d) LSTCA, (e) tLASCA, (f) sLASCA, (g) stLASCA, (h) aLASCA, (i) gLASCA methods (top: $m=5$; bottom: $m=25$ ). 
larger spatial window $(N s)$ except for LSTCA method. ${ }^{23}$

The contrast images with LSSCA, LSTCA, tLASCA, sLASCA, stLASCA, aLASCA and gLASCA methods for the simulated data were shown in Figs. 4(c)-4(i). We hypothesized that the intensity image shown in Fig. 4(a) of scattering surface was cerebrovascular image where dark regions of radial shape represented the blood vessels. Arbitrarily, the intensity of radial line region was 0.5 and the background region was 0.9 . As mentioned above, the parameter $m$ in Eq. (1) affected the correlation time $\tau$. Therefore, we simulated a series of flow velocity of dynamic speckle images by changing parameter $m$ from 5 to 25 in the radial line region ( 30 frames). For example, the speckle image of $m=25$ was shown in Fig. 4(b) and the contrast images of $m=5$ and $m=25$ were shown in Figs. 4(c)-4(i). The intensity of radial line region showed higher value in the first row $(m=5)$ than that in the second row $(m=25)$, which illustrated that a smaller $m$ corresponds to a smaller $1 / \tau$, as well as a faster spatial blurring of the speckle pattern. gLASCA method also complied with this theory. Besides, Fig. 4(h) showed more spurs than Fig. 4(i) along the edge of radial lines.

The contrast images calculated from mice brain vessels were shown in Fig. 5. The field of view was approximately $2.5 \times 2.5 \mathrm{~mm}$. The size of each raw speckle image was $512 \times 512$ pixels. According to the principle of the LSCI, the resolution of the image was $4.88 \mu \mathrm{m}$. gLASCA produced a significant improvement in micro vessel distinguishability of high resolution especially under the condition where vessels has a low flow velocity in red square ROIs of Fig. 5. These microvessels were indistinguishable in both LSSCA and LSTCA images shown in Figs. 5(a) and 5(b). Meanwhile, those images calculated using spatiotemporal methods shown in Figs. 5(c)-5(e) show vague and small graininess. The vessels were not like the continuous lines, but rather as sprays of dots. Compared with the aLASCA, gLASCA image displayed better contrast between micro vessels and tissues.

Furthermore, the global contrast value using spatial methods was higher than that by using temporal methods in CBF images, which was (a)
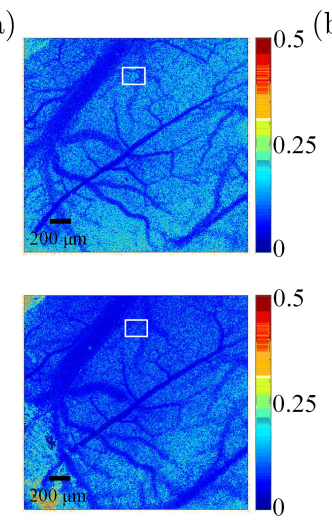

(e)
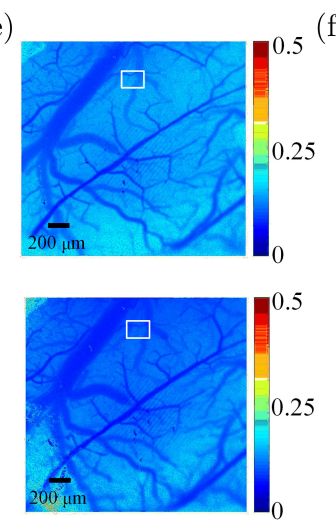
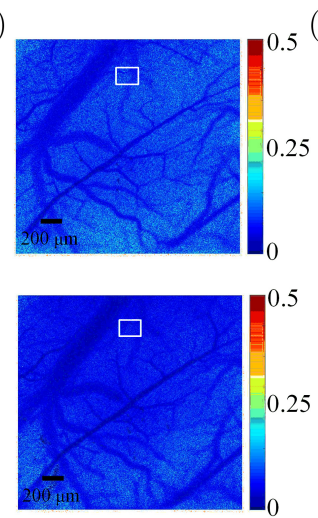

(f)
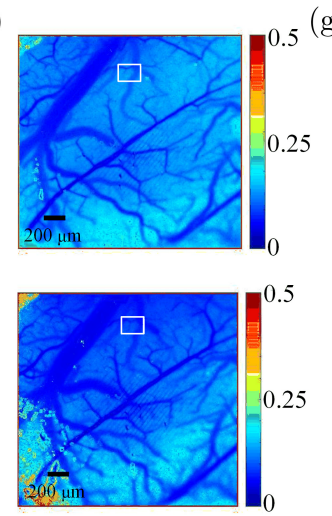

(c)
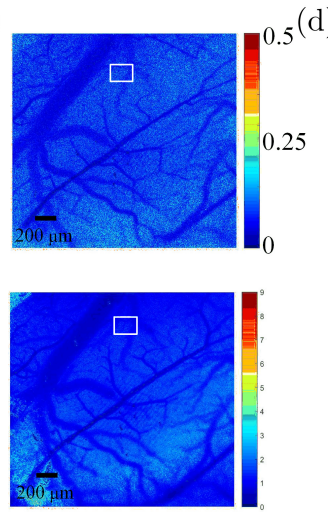

g)
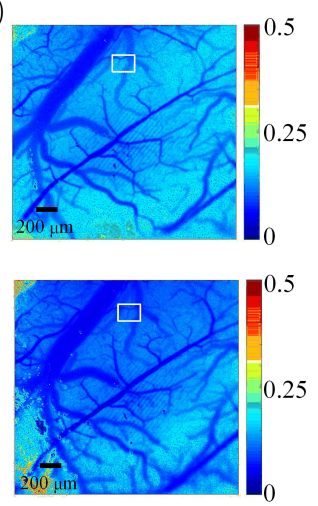

Fig. 5. Comparisons between contrast images computed from mice brain vessels using (a) LSSCA, (b) LSTCA, (c) tLASCA, (d) sLASCA, (e) stLASCA, (f) aLASCA, (g) gLASCA methods (top: $t=0 \mathrm{~min}$; bottom: $t=70 \mathrm{~min}$ ). 
similar in the simulation of blood images. Interestingly, we also witnessed that the intensity of cerebral vascular region was higher in the first row $(t=0 \mathrm{~min})$ than that in the second row $(t=$ $70 \mathrm{~min}$ ), which illustrated the dynamic variation of cortical blood flow pre- and pose the reference point was in accordance with that of the simulation model. In other words, the increasing parameter $m$ was associated with the fast-moving particles, so it directly showed the CBF increased after the reference node of perfusion.

In Figs. 6(a) and 6(b), we calculated all the mean contrast values of 25 regions of interest (ROI), such as the ones highlighted by rectangle in Fig. 5, in vascular region with methods of LSSCA, LSTCA, tLASCA, sLASCA, stLASCA and gLASCA. In Fig. 6(a), all the mean contrast $K$ values kept a fall trend when the parameter $m$ increased gradually with the interval of 5 . While the mean contrast $K$ value increased slowly before reference point and dropped substantially after reference node in animal perfusion models, notably the time point 20 was the reference point of the beginning of perfusion shown in Fig. 6(b). The tendency of speckle contrast $K$ became highly homogeneous in simulation and experimental mouse model. We could observe that the mean contrast $K$ value obtained by stLASCA was the largest among the others.

Meanwhile, we calculated the CNR of 25 ROIs to evaluate the quality of LSCI images with different algorithms. The value of CNR was defined as a distinguishability between the $\mathrm{CBF}$ and the background. We found that gLASCA method showed best accuracy in representing flow velocity levels of distinguishable and lowest noise in simulation and experiment models in Figs. 6(c) and 6(d). gLASCA method showed $10.2 \%(p<0.01, t$ test $)$ and $7.1 \%$ ( $p<0.05, t$ test $)$ higher contrast-to-noise

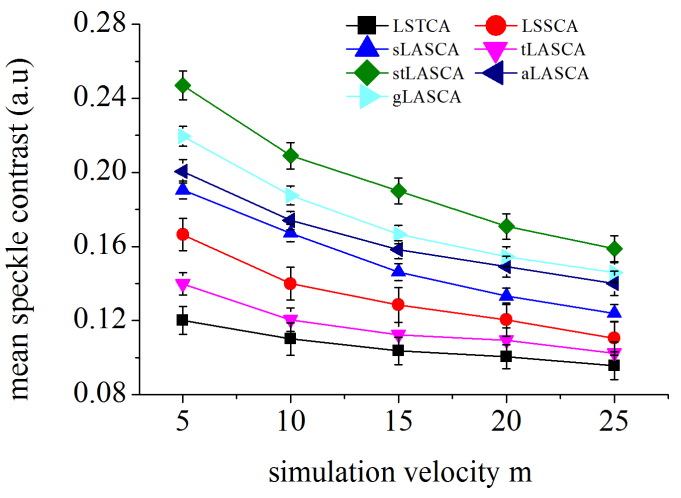

(a)

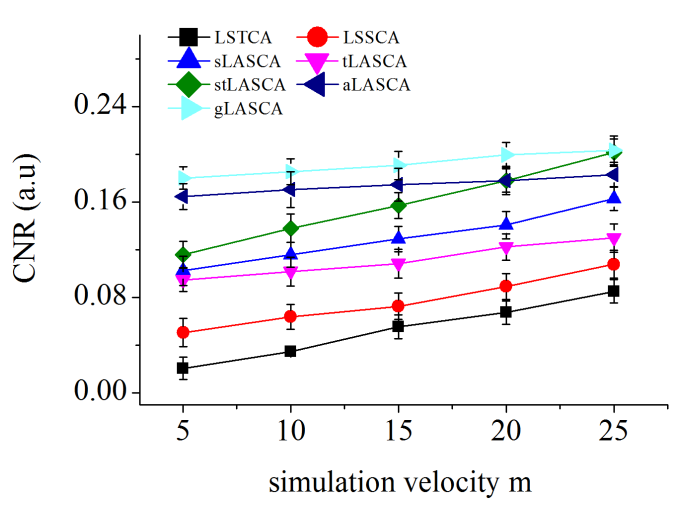

(c)

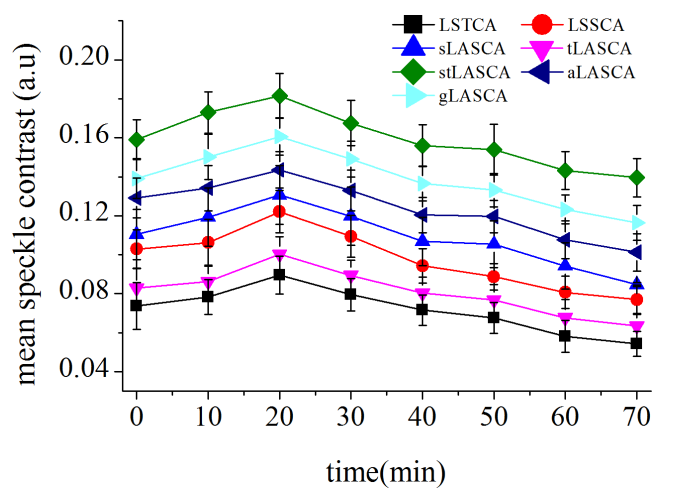

(b)

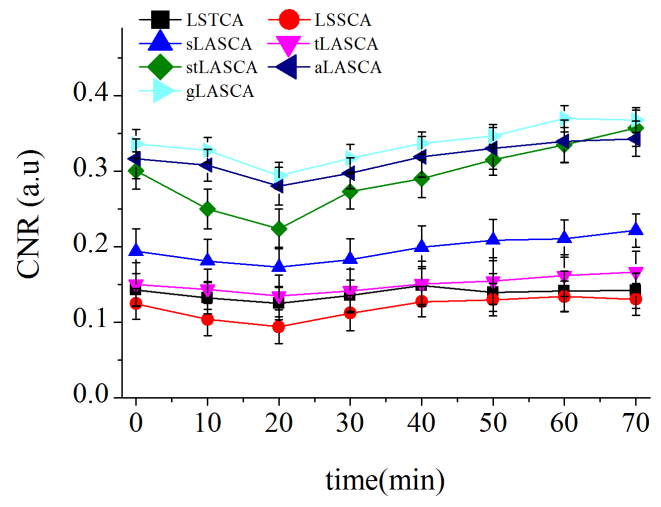

(d)

Fig. 6. Speckle contrast analysis of simulated dynamic speckle and mice cerebral blood vessels. Changes of (a) mean speckle contrast and (c) contrast to noise (CNR) with $m$ in Eq. (1) by using 6 algorithms. Changes of (b) mean speckle contrast and (d) contrast to noise (CNR) pre- and pose the typical local perfusion by using 6 algorithms. (notably the time point 20 is the reference node of the beginning of perfusion). 
(a)

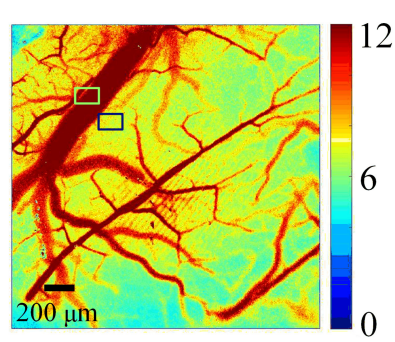

(d)

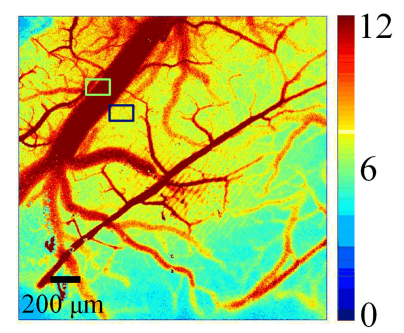

(g)

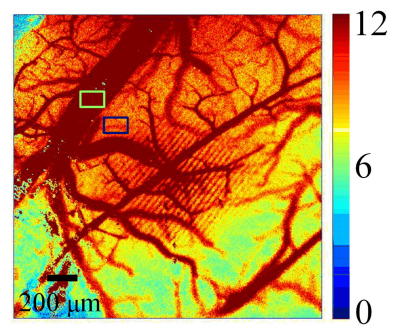

(b)

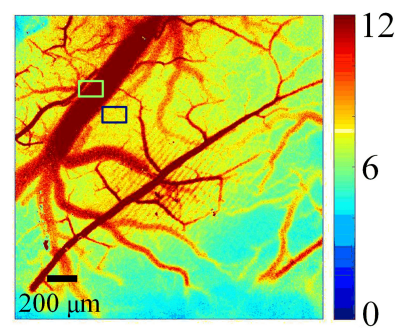

(e)

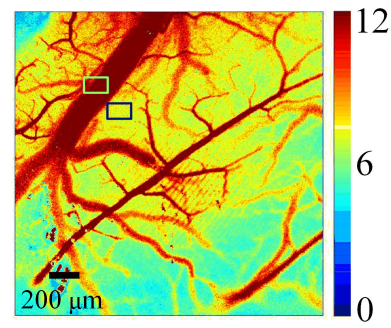

(h)

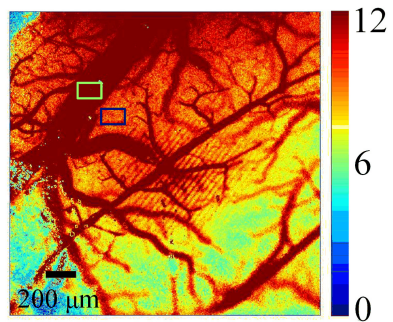

(c)

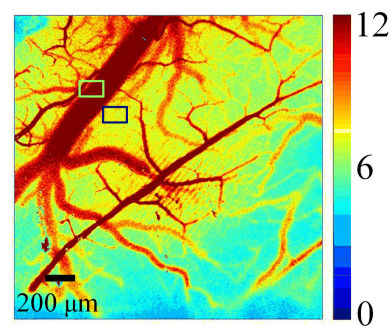

(f)

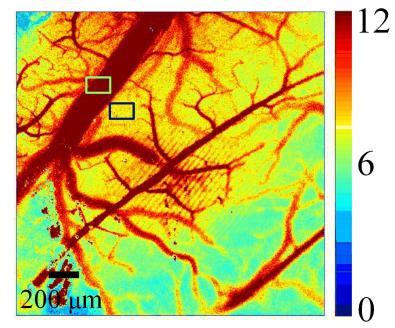

(i)

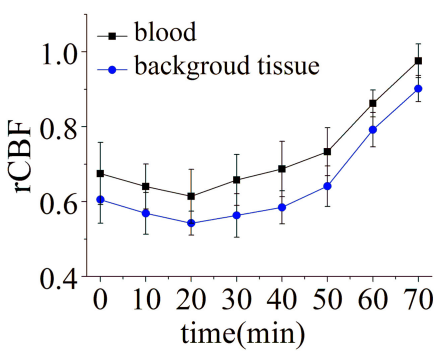

Fig. 7. Relative CBF changes of laser speckle images of local perfusion mice at each time point including (a),(b), (c), (d) (e), (f),(g) and (h), notably the time point (c) is the reference node of the beginning of perfusion. (i) Normalized relative CBF and background tissue of the ROI calculated using LLSCI.

ratio (CNR) both in the simulation and experiment models compared to aLASCA.

Figure 7 represented the CBF image of the cranial window of cortex for the mice, before and after the perfusion. We noted that the CBF change of pos-perfusion changes circled by gray rectangle were substantially higher than that of pre-perfusion, which was consistent with variation of the tissue background in blue rectangular box. Moreover, we could find a surge pos- $20 \mathrm{~min}$ at the reference point, which was in accordance with transient cerebral ischemia reperfusion physiological mechanism shown in Fig. 7(i). Therefore, it is very important to monitor the microcirculation tissue perfusion.

gLASCA is an algorithm to process speckle data preferentially along the flow direction. In microvessels, the direction of minimum contrast is along radially the blood vessels. Therefore, we can obtain the direction of blood flow as long as the direction of speckle contrast selected is the minimum. Thus, we proved that the present gLASCA algorithm brain had a significant resolution improvement in microvasculature over current techniques imaging of mouse.

\section{Discussion}

In the present study, the method of gLASCA processed laser speckle image with the preferred convolutional mark, which aligns with the vascular flow direction. This algorithm just required three raw spectral image frames for contrast calculation, which provided a superiority in temporal resolution of cerebrovascular imaging. Compared with other spatiotemporal algorithms, the contrast images obtained by using gLASCA method showed a margin increase in CNR of ROIs.

Since segments kernel selected along the direction of each $15^{\circ}$ angle were found to respond well, 12 different templates were applied in the our 
proposed method to search for the optimal direction operator along all possible orientations. ${ }^{24}$ Thus, applications of the designed Gaussian operator enhanced $K$ value discrepancy in the anisotropic method based on speckle image. Meanwhile, a proper convolutional mark along the direction of the minimal $K$ value could be applied in refresh a high-quality contrast image in anistropic method, especially in the condition of slower flow velocity as shown in Figs. 4 and 5. Selecting parameter $\sigma$ as a smaller value would match well with cerebral microvessels of medium caliber for the cerebral images acquired by our experiment equipment. ${ }^{25}$

Unlike LSTCA with the characteristics of highest spatial pixel resolution, all other spatial or spatiotemporal algorithms sacrifices the spatial resolution with a minimum of $3 \times 3$ pixels in simulation models, as well as $5 \times 5$ pixels in experiment models. Of note, gLASCA has a spatial resolution of Gaussian matched operator where the mask is the resolution match the vessel axis shape. Furthermore, gLASCA has a better performance in contrast with the other algorithms to assess the relative changes for CNR. We observe that the spatial algorithm reacts better to the static object of the scattered source, whereas the temporal algorithm reacts more promptly to the dynamic object of the scattered source. ${ }^{26}$ In clinical trials, blood flow in human tissue would change over time, so did laser speckle image system. Thus, our algorithm can adapt itself to improve accuracy along the orientation of blood flow.

We have explored the superiority and feasibility of gLASCA to employ in mice perfusion studies. Despite pulsatility of blood flow induced by heart rate should keep the frequency of $2.25 \mathrm{~Hz}$ to $3 \mathrm{~Hz}$, tLASCA just required a temporal window of 40 frames in total of $1.6 \mathrm{~s}$, given the camera speed (45 frames/s). Therefore, gLASCA with the temporal convolution window can apply in less frames per second to monitor the perfusion response. It is a recommended technique for scientists to research the animal models of cerebrovascular reactivity.

\section{Acknowledgments}

This study was supported by National Major Scientific Instruments and Equipments Development Project Funded by National Natural Science Foundation of China (81827803, 81727804),
National Natural Science Foundation of China (61875085, 81601532), Natural Science Foundation of Jiangsu Province (BK20160814), Jiangsu Science and Technology Support Plan (Social Development) (BE2016759), and Jiangsu Innovation Program for Graduate Education (KYLX16_339). All authors declare that the research was conducted in the absence of any commercial or financial relationships that could be construed as a potential conflict of interest.

\section{References}

1. D. A. Boas, A. K. Dunn, "Laser speckle contrast imaging in biomedical optics," J. Biomed. Optics 15(1), 011109 (2000).

2. A. Humeau-Heurtier et al., "Relevance of laser doppler and laser speckle techniques for assessing vascular function: State of the art and future trends," IEEE Trans. Biomed. Eng. 60(3), 659-666 (2013).

3. K. Murari et al., "Contrast-enhanced imaging of cerebral vasculature with laser speckle," Appl. Optics 46(22), 5340-5346 (2007).

4. D. Briers et al., "Laser speckle contrast imaging: Theoretical and practical limitations," J. Biomed. Optics 18(6), 66018 (2013).

5. A. Ponticorvo et al., "Laser speckle contrast imaging of blood flow in rat retinas using an endoscope," J. Biomed. Optics 18(9), 090501 (2013).

6. S. Mori, J. Zhang, "Principles of diffusion tensor imaging and its applications to basic neuroscience research," Neuron 51(5), 527-539 (2006).

7. H. K. Shin et al., "Normobaric hyperoxia improves cerebral blood flow and oxygenation, and inhibits peri-infarct depolarizations in experimental focal ischaemia," Brain 130(6), 1631 (2007).

8. A. F. Fercher and J. D. Briers, "Flow visualization by means of single-exposure speckle photography," Opt. Commun. 37(5), 326-330 (1981).

9. H. Cheng et al., "Modified laser speckle imaging method with improved spatial resolution," J. Biomed. Optics 8(3), 559-564 (2003).

10. J. D. Briers, S. Webster, "Laser speckle contrast analysis (lasca): A nonscanning, full-field technique for monitoring capillary blood flow," J. Biomed. Optics 1(2), 174-179 (1996).

11. T. M. Le et al., "New insights into image processing of cortical blood flow monitors using laser speckle imaging," IEEE Trans. Med. Imaging 26(6), 833-842 (2007).

12. J. Qiu et al., Spatiotemporal laser speckle contrast analysis for blood flow imaging with maximized speckle contrast," J. Biomed. Optics 15(1), 016003 (2010). 
13. A. Rege et al., "Anisotropic processing of laser speckle images improves spatiotemporal resolution," IEEE Trans. Biomed. Eng. 59(5), 1272-1280 (2012).

14. D. D. Duncan, S. J. Kirkpatrick, "The copula: A tool for simulating speckle dynamics," J. Opt. Soc. America A Optics Image Sci. Vis. 25(1), 231-237 (2008).

15. S. J. Kirkpatrick et al., "Detrimental effects of speckle-pixel size matching in laser speckle contrast imaging," Opt. Lett. 33(24), 2886-2888 (2008).

16. D. Clark et al., "Impact of aging on spreading depolarizations induced by focal brain ischemia in rats," Neurobiol. Aging 35(12), 2803 (2014).

17. U. T. V. Nguyen, A. Bhuiyan, L. A. F. Park, et al., "An effective retinal blood vessel segmentation method using multi-scale line detection," Pattern Recognit. 46(3), 703-715 (2013).

18. D. Marin et al., "A new supervised method for blood vessel segmentation in retinal images by using gray-level and moment invariants-based features," IEEE Trans. Med. Imaging 30(1), 146 (2011).

19. E. Ricci, R. Perfetti, "Retinal blood vessel segmentation using line operators and support vector classification," IEEE Trans. Med. Imaging 26(10), 1357-1365 (2007).
20. L. Gang et al., "Detection and measurement of retinal vessels in fundus images using amplitude modified second-order gaussian filter," IEEE Trans. Biomed. Eng. 49(2), 168-172 (2002).

21. B. Liu, "Research methods of retinal vessels segmentation," Doctoral Dissertation, Central South University (2011).

22. W. Marijke, R. Yves, "On the definition of signalto-noise ratio and contrast-to-noise ratio for fmri data," Plos One, 8(11), e77089 (2013).

23. S. M. S. Kazmi et al., "Expanding applications, accuracy, and interpretation of laser speckle contrast imaging of cerebral blood flow," J. Cereb. Blood Flow Metab. 35(7), 1076-1084 (2015).

24. J. Zhang et al., "A retinal vessel boundary tracking method based on Bayesian theory and multi-scale line detection," Computerized Medical Imaging and Graphics, 38(6), 517-525 (2014).

25. R. Zwiggelaar et al., "Linear structures in mammographic images: Detection and classification," IEEE Trans. Med. Imaging 23(9), 1077-1086 (2004).

26. D. D. Duncan et al., "Statistics of local speckle contrast," J. Opt. Soc. America Opt. Image Sci. Vis. 25(1), 9-15 (2008). 